\title{
Intensive Column Apparatus for Chemical Reactions
}

\author{
Chr. Boyadjiev*, M. Doichinova, P. Popova-Krumova, B. Boyadjiev \\ Institute of Chemical Engineering, Bulgarian Academy of Sciences, Sofia, Bulgaria \\ Email: ${ }^{*}$ chr.boyadjiev@gmail.com
}

Received 6 April 2014; revised 20 May 2014; accepted 28 May 2014

Copyright $@ 2014$ by authors and OALib.

This work is licensed under the Creative Commons Attribution International License (CC BY). http://creativecommons.org/licenses/by/4.0/

(c) (i) Open Access

\begin{abstract}
A solution of the intensification problem of the column chemical reactors is presented in the approximation of the mechanics of continua. The effect of the radial non-uniformity of the velocity distribution, the effect of the tangential flow and simultaneous mass, and heat transfer processes are analyzed. A theoretical analysis of the simultaneous mass and heat transfer processes in column reactors is presented.
\end{abstract}

\section{Keywords}

Intensification, Column Reactor, Radial Non-Uniformity, Tangential Flow, Mass Transfer, Heat Transfer

\section{Introduction}

A fundamental problem in the column chemical reactors is the radial non-uniformity of the axial velocity distribution. The theoretical analysis of this problem is possible to be made, using a new approach on the base of the approximations of the mechanics of continua [1]-[4], where the mathematical point is equivalent to a small (elementary) physical volume, which is sufficiently small with respect to the apparatus volume, but at the same time sufficiently large with respect to the intermolecular volumes of the medium. As a result the mathematical description of the processes is mass balance in this elementary volume in the form of a convection-diffusion type of models, using the convection-diffusion equations. These models [1]-[4] permit to be made a qualitative analysis of the process for to be obtained the main, small and slight physical effects (mathematical operators in the models), and to be rejected the slight effects (operators).

The using of the convection-diffusion type of models for a quantitative analysis of the processes in column apparatuses is not possible because the velocity function in the convection-diffusion equations is unknown. The

${ }^{*}$ Corresponding author.

How to cite this paper: Boyadjiev, Chr., et al. (2014) Intensive Column Apparatus for Chemical Reactions. Open Access Library Journal, 1: e413. http://dx.doi.org/10.4236/oalib.1100413 
problem can be avoided if the average values of the velocities and concentrations over the cross-sectional area of the columns are used, i.e. the medium elementary volume (in the physical approximations of the mechanics of continua [1]-[4]) will be equivalent to a small cylinder with radius $r_{0}$ and a height, which is sufficiently small with respect to the column height and at the same time sufficiently large with respect to the intermolecular distances in the medium.

A main problem in the column chemical reactors is the decrease of the processes efficiency as a result of the effect of the radial non-uniformity of the axial velocity distribution. This problem can be avoided using a tangential inlet of the flow in the column, which is very useful in the cases of simultaneous mass and heat transfer processes.

\section{Chemical Reactor Modeling}

Let's consider a liquid (gas) motion in a column reactor with radius $r_{0}(\mathrm{~m})$ and height $l(\mathrm{~m})$, where a homogeneous chemical reaction between two fluid components is realized. If the difference between the component concentrations is very large, then the chemical reaction will be first order.

\subsection{Convection-Diffusion Type of Model}

If the velocity $u\left(\mathrm{~m} \cdot \mathrm{s}^{-1}\right)$ and concentration $c\left(\mathrm{~kg} \cdot \mathrm{m}^{-3}\right)$ distributions in the column are defined as:

$$
u=u(r), \quad c=c(r, z),
$$

the convection-diffusion type of model [4] can be expressed as:

$$
\begin{aligned}
& u \frac{\partial c}{\partial z}=D\left(\frac{\partial^{2} c}{\partial z^{2}}+\frac{1}{r} \frac{\partial c}{\partial r}+\frac{\partial^{2} c}{\partial r^{2}}\right)-k c \\
& z=0, \quad c(r, 0) \equiv c_{0}, \quad \bar{u} c_{0} \equiv u c_{0}-D \frac{\partial c}{\partial z} \\
& r=0, \quad \frac{\partial c}{\partial r} \equiv 0 ; \quad r=r_{0}, \quad \frac{\partial c}{\partial r} \equiv 0,
\end{aligned}
$$

where $D\left(\mathrm{~m}^{2} \cdot \mathrm{s}^{-1}\right)$ is diffusivity, $k\left(\mathrm{~s}^{-1}\right)$-chemical reaction rate constant, $\bar{u}, c_{0}$-input values of the average velocity and concentration.

The mass transfer efficiency $(g)$ in the column and conversion degree $(G)$ is possible to be obtained [4], using the input and outlet average convective mass fluxes at the cross-sectional area surface in the column:

$$
g=\bar{u} c_{0}-\frac{2}{r_{0}^{2}} \int_{0}^{r_{0}} r u c(r, l) \mathrm{d} r, \quad G=\frac{g}{\bar{u} c_{0}} .
$$

The qualitative analysis of the model (2) will be made, using generalized variables:

$$
\begin{aligned}
& r=r_{0} R, \quad z=l Z, \quad u(r)=u\left(r_{0} R\right)=\bar{u} U(R), \\
& c(r, z)=c\left(r_{0} R, l Z\right)=c_{0} C(R, Z), \quad \varepsilon=\left(\frac{r_{0}}{l}\right)^{2},
\end{aligned}
$$

where $r_{0}, l, \bar{u}, c_{0}$ are the characteristic (inherent) scales (maximal or average values) of the variables. The introducing of the generalized variables (4) in (2) leads to:

$$
\begin{aligned}
& U(R) \frac{\partial C}{\partial Z}=\mathrm{Fo}\left(\varepsilon \frac{\partial^{2} C}{\partial Z^{2}}+\frac{1}{R} \frac{\partial C}{\partial R}+\frac{\partial^{2} C}{\partial R^{2}}\right)-\mathrm{Da} C \\
& Z=0, \quad C \equiv 1, \quad 1 \equiv U-\mathrm{Pe}^{-1} \frac{\partial C}{\partial Z} \\
& R=0, \quad \frac{\partial C}{\partial R} \equiv 0 ; \quad R=1, \quad \frac{\partial C}{\partial R} \equiv 0,
\end{aligned}
$$


where $\varepsilon=\mathrm{Fo}^{-1} \mathrm{Pe}^{-1}, \mathrm{Fo}=\frac{D l}{\bar{u} r_{0}^{2}}, \mathrm{Pe}=\frac{\bar{u} l}{D}, \mathrm{Da}=\frac{k l}{\bar{u}}$ are the Fourier, Damkohler and Peclet numbers, respectively.

In the cases of big values of the average velocity $\left(0=\mathrm{Fo} \leq 10^{-2}\right)$, from the convection-diffusion type of model (5) is possible to be obtained a convection type of model if put $\mathrm{Fo}=0$ :

$$
U(R) \frac{\partial C}{\partial Z}=-\mathrm{DaC} ; \quad Z=0, \quad C \equiv 1 .
$$

The effect of the chemical reaction rate is negligible if $0=\mathrm{Da} \leq 10^{-2}$ and as a result $C \equiv 1$.

When a fast chemical reactions take place $\left(\mathrm{Da} \geq 10^{2}\right)$, the terms in the model must be divided by $\mathrm{Da}$ and the approximation $0=\mathrm{Da}^{-1} \leq 10^{-2}$ has to be applied and as a result:

$$
0=\frac{\mathrm{Fo}}{\mathrm{Da}}\left(\frac{1}{R} \frac{\mathrm{d} C}{\mathrm{~d} R}+\frac{\mathrm{d}^{2} C}{\mathrm{~d} R^{2}}\right)-C ; \quad R=0, \frac{\mathrm{d} C}{\mathrm{~d} R}=0 ; \quad R=1, \frac{\mathrm{d} C}{\mathrm{~d} R}=0,
$$

i.e. the model (6) is diffusion type.

\subsection{Average Concentration Model}

The average velocity and concentration at the column cross-sectional area can be presented as

$$
\bar{u}=\frac{2}{r_{0}^{2}} \int_{0}^{r_{0}} r u(r) \mathrm{d} r, \quad \bar{c}(z)=\frac{2}{r_{0}^{2}} \int_{0}^{r_{0}} r c(r, z) \mathrm{d} r .
$$

The velocities and concentration distributions in the convection-diffusion type of model (2) assume to be presented [3] [4] by the average functions (8):

$$
u(r)=\bar{u} \tilde{u}(r), \quad c(r, z)=\bar{c}(z) \tilde{c}(r, z),
$$

where $\tilde{u}(r)$ and $\tilde{c}(r, z)$ represent the radial nonuniformity of both the velocity and the concentration distributions, satisfying the conditions:

$$
\frac{2}{r_{0}^{2}} \int_{0}^{r_{0}} r \tilde{u}(r) \mathrm{d} r=1, \quad \frac{2}{r_{0}^{2}} \int_{0}^{r_{0}} r \tilde{c}(r, z) \mathrm{d} r=1
$$

An average concentration model may be obtained [1]-[4] if put the Expression (9) into the model Equation (2) and then multiply by $r$ and integrate with respect to $r$ over the interval $\left[0, r_{0}\right]$. As a result:

$$
\alpha \bar{u} \frac{\partial \bar{c}}{\partial z}+\frac{\mathrm{d} \alpha}{\mathrm{d} z} \overline{u c}=D \frac{\partial^{2} \bar{c}}{\partial \mathrm{z}^{2}}-k \bar{c} ; \quad z=0, \quad \bar{c}(0)=c_{0}, \quad \frac{\partial \bar{c}}{\partial z}=0,
$$

where

$$
\alpha=\alpha(z)=\frac{2}{r_{0}^{2}} \int_{0}^{r_{0}} r \tilde{u} \tilde{c} \mathrm{~d} r
$$

A using of the generalized variables:

$$
Z=\frac{Z}{l}, \quad \bar{C}=\frac{\bar{c}}{c_{0}}, \quad \alpha(z)=\alpha(l Z)=A(Z),
$$

leads to:

$$
A \frac{\mathrm{d} \bar{C}}{\mathrm{~d} Z}+\frac{\mathrm{d} A}{\mathrm{~d} Z} \bar{C}=\mathrm{Pe}^{-1} \frac{\mathrm{d}^{2} \bar{C}}{\mathrm{~d} Z^{2}}-\mathrm{Da} \bar{C} ; \quad Z=0, \quad \bar{C}=1, \quad \frac{\mathrm{d} \bar{C}}{\mathrm{~d} Z}=0 .
$$

In the cases $0=\mathrm{Fo} \leq 10^{-2}, 0=\mathrm{Pe}^{-1}=\varepsilon \mathrm{Fo} \leq 10^{-2}, \varepsilon<1$ (see (6)) the model (14) has the convective form:

$$
A \frac{\mathrm{d} \bar{C}}{\mathrm{~d} Z}+\frac{\mathrm{d} A}{\mathrm{~d} Z} \bar{C}=-\mathrm{Da} \bar{C} ; \quad Z=0, \quad \bar{C}=1 .
$$


The function $A(Z)$ in (14) and (15) represents the effect of the velocity radial non-uniformity on the mass transfer efficiency in the column apparatus:

$$
A(Z)=\alpha(z)=\frac{2}{r_{0}^{2}} \int_{0}^{r_{0}} r \tilde{c} \tilde{d} \mathrm{~d} r
$$

where

$$
\begin{aligned}
& \tilde{u}(r)=\frac{u(r)}{\bar{u}}=U(R), \\
& \tilde{c}(r, z)=\frac{c(r, z)}{\bar{c}(z)}=\frac{C(R, Z)}{\bar{C}(Z)}, \\
& \bar{C}(Z)=\frac{\bar{c}(z)}{c_{0}}=2 \int_{0}^{1} R C(R, Z) \mathrm{d} R
\end{aligned}
$$

and as a result:

$$
A(Z)=2 \int_{0}^{1} R U(R) \frac{C(R, Z)}{\bar{C}(Z)} \mathrm{d} R
$$

\subsection{Effect of the Radial Non-Uniformity of the Velocity Distribution}

As an example will be used the case of parabolic velocity distribution (Poiseuille flow):

$$
u(r)=\bar{u}\left(2-2 \frac{r^{2}}{r_{o}^{2}}\right)
$$

From (4) and (19) follows:

$$
U(R)=2-2 R^{2} .
$$

The solutions of the problems (5) for $\mathrm{Da}=1,2$ and $\mathrm{Fo}=0,0.1,1.0$ permits to be obtained $C(R, Z), \bar{C}(Z)$ and results for $A(Z) \geq 1$ are presented, using different approximations:

$$
\begin{aligned}
& \text { Fo }=1, \quad \mathrm{Da}=1,2, \quad A(Z)=a_{0}, \quad a_{0}=\frac{1}{N} \sum_{n=1}^{N} A\left(Z_{n}\right) ; \\
& \text { Fo }=0,0.1, \quad \mathrm{Da}=1,2, \quad A(Z)=1+a Z, \quad a=\frac{1}{N} \sum_{n=1}^{N} \frac{A\left(Z_{n}\right)-1}{Z_{n}} ; \quad 0<Z_{n}<1 .
\end{aligned}
$$

The obtained values of $a, a_{0}$ in (21) are shown in Table 1 .

The Equations (14) and (15) permit to be obtained expressions for the concentration axial gradient:

$$
\begin{aligned}
& \frac{\mathrm{d} \bar{C}}{\mathrm{~d} Z}=-A^{-1} \frac{\mathrm{d} A}{\mathrm{~d} Z} \bar{C}+A^{-1} \mathrm{Pe}^{-1} \frac{\mathrm{d}^{2} \bar{C}}{\mathrm{~d} Z^{2}}-A^{-1} \mathrm{Da} \bar{C} ; \\
& \frac{\mathrm{d} \bar{C}}{\mathrm{~d} Z}=-A^{-1} \frac{\mathrm{d} A}{\mathrm{~d} Z} \bar{C}-A^{-1} \mathrm{Da} \bar{C} .
\end{aligned}
$$

Table 1. Parameters values and values of process efficiencies (conversion degree).

\begin{tabular}{ccccc}
\hline & $a$ & $a_{0}$ & $G$ & $G_{0}$ \\
$\mathrm{Da}=1, \mathrm{Fo}=0$ & 0.5511 & & 0.5568 & 0.6734 \\
$\mathrm{Da}=1, \mathrm{Fo}=0.1$ & 0.2463 & & 0.5938 & 0.6452 \\
$\mathrm{Da}=$ 1, Fo $=1$ & & 1.02 & 0.6211 & 0.6281 \\
$\mathrm{Da}=$ 2, Fo $=0$ & & 0.7806 & 0.8516 \\
$\mathrm{Da}=$ 2, Fo $=0.1$ & 1.3623 & & 0.8115 & 0.8502 \\
$\mathrm{Da}=$ 2, Fo $=1$ & 0.4547 & 1.04 & 0.8481 & 0.8538 \\
\hline
\end{tabular}


From (9) it follows, that $\tilde{u}=1$ if the velocity radial non-uniformity is absent $(u=\bar{u})$, i.e. $A=\alpha=1$ (see ((10), (12), (16)). The presence of a radial non-uniformity of the axial velocity in the columns leads to $A>1$, i.e. a decrease of the concentration axial gradient and process efficiency [4]. The using ((3), (4), (13)) permits to be obtained the process efficiencies (conversion degree) in the cases of presence $(G)$ and absence $\left(G_{0}\right)$ of a radial non-uniformity of the axial velocity in the column:

$$
G=1-2 \int_{0}^{1} R U(R) C(R, 1) \mathrm{d} R, \quad G_{0}=1-\bar{C}(1) .
$$

The results are shown in the Table 1 , where is seen, that the radial non-uniformity of the axial velocity component leads to a substantially decrease of the conversion degree.

\subsection{Effect of the Tangential Flow}

Let's consider a cylindrical column with axial input of the gas (liquid) flow (Figure 1(a)). The axial and radial velocity components $u_{z}=u_{z}(r, z), u_{r}=u_{r}(r, z)$ satisfy the continuity equation:

$$
\frac{\partial u_{z}}{\partial z}+\frac{\partial u_{r}}{\partial r}+\frac{u_{r}}{r}=0, \quad z=0, \quad u_{z}(r, 0)=u_{z}^{0}(r), \quad r=r_{0}, \quad u_{r}\left(r_{0}, z\right) \equiv 0,
$$

where $u_{z}^{0}(r)$ is the input distribution of the axial velocity component and is a result of the geometric conditions at the axial input of the column. The velocity components $u_{z}(r, z), u_{r}(r, z)$ are possible to be obtained as a solution of the Navier-Stokes equations in boundary layer approximation, i.e. to solve the problem of the gas (liquid) jet in immobile gas (liquid). As a result the radial non-uniformity of the axial velocity component exists for the columns with limit height. In these conditions the conversion degree increase is related with the decrease of the radial non-uniformity of the axial velocity component (special geometric conditions at the axial input of the column).

A possibility for a partial reduction of the radial non-uniformity of the axial velocity component is the using of a column with tangential enter [5] of the gas (liquid) flow (Figure 1(b)) in the column input.

A maximal reduction of the radial nonuniformity of the axial velocity component is the using of a column with tangential enter [6] of the gas (liquid) flow (Figure 1(c)) in the column working area.

In the cases of tangential input of the flow in the column, the velocity components $u_{z}=u_{z}(z, r, \varphi), u_{r}=u_{r}(z, r, \varphi), u_{\varphi}(z, r, \varphi)$ satisfy the continuity equation:

$$
\begin{aligned}
& \frac{\partial u_{z}}{\partial z}+\frac{1}{r} \frac{\partial u_{\varphi}}{\partial \varphi}+\frac{\partial u_{r}}{\partial r}+\frac{u_{r}}{r}=0 \\
& z=0, \quad 0 \leq r<r_{0}, \quad 0 \leq \varphi \leq 2 \pi, \quad u_{z}(0, r, \varphi) \equiv \bar{u}=\frac{Q}{\pi r_{0}^{2}} \\
& r=r_{0}, \quad 0<z \leq l, \quad 0 \leq \varphi \leq 2 \pi, \quad u_{r}\left(z, r_{0}, \varphi\right) \equiv 0 \\
& \varphi=0, \quad u_{\varphi}\left(0, r_{0}, 0\right) \equiv u_{\varphi}^{0}=\frac{Q}{\pi r_{00}^{2}},
\end{aligned}
$$

where $Q\left(\mathrm{~m}^{3} \cdot \mathrm{s}^{-1}\right)$ is gas (liquid) flow rate in the column, $r_{00}$ —column input radius.

The using of the generalized variables:

$$
z=l Z, \quad r=r_{0} R, \quad \varphi=2 \pi \Phi, \quad u_{z}=\bar{u} U_{z}, \quad u_{r}=\bar{u} \frac{r_{0}}{l} U_{r}, \quad u_{\varphi}=u_{\varphi}^{0} U_{\varphi},
$$

leads to

$$
\begin{aligned}
& \frac{1}{R} \frac{\partial U_{\varphi}}{\partial \varphi}+2 \pi \frac{\bar{u} r_{0}}{u_{\varphi}^{0} l}\left(\frac{\partial U_{z}}{\partial Z}+\frac{\partial U_{r}}{\partial R}+\frac{U_{r}}{R}\right)=0 \\
& Z=0, \quad 0 \leq R<1, \quad 0 \leq \Phi \leq 1, \quad U_{z}(0, R, \Phi) \equiv 1 \\
& R=1, \quad 0<Z \leq 1, \quad 0 \leq \Phi \leq 1, \quad U_{r}(Z, 1, \Phi) \equiv 0 \\
& \Phi=0, \quad U_{\varphi}(0,1,0) \equiv 1 .
\end{aligned}
$$




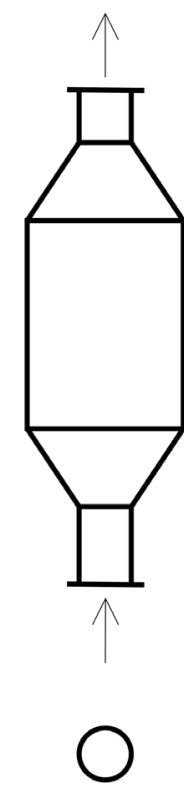

(a)

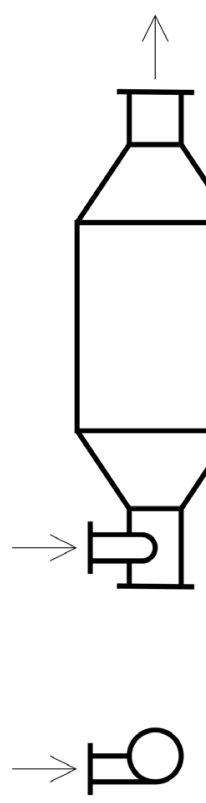

(b)

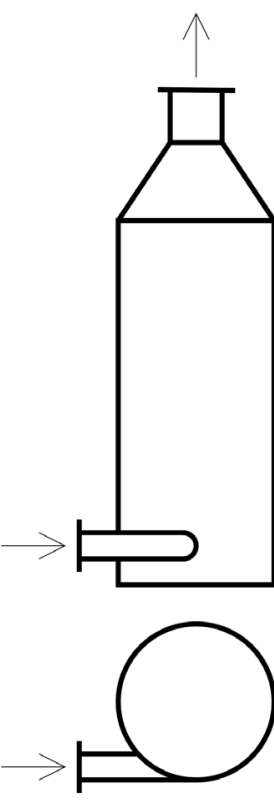

(c)

Figure 1. Cylindrical column with: (a) Axial gas (liquid) flow; (b) Tangential gas (liquid) flow in column input; (c) Tangential gas (liquid) flow in column working area.

Practically $\bar{u} \ll u_{\varphi}^{0}$ and an approximation is possible to be used:

$$
0=2 \pi \frac{\bar{u} r_{0}}{u_{\varphi}^{0} l} \leq 10^{-2},
$$

i.e.

and from (27) it follows:

$$
\frac{\partial U_{\varphi}}{\partial \varphi}=0
$$

$$
\begin{aligned}
& \frac{\partial U_{z}}{\partial Z}+\frac{\partial U_{r}}{\partial R}+\frac{U_{r}}{R}=0 ; \\
& Z=0, \quad 0 \leq R<1, \quad U_{z}(0, R) \equiv 1 ; \\
& R=1, \quad 0<Z \leq 1, \quad U_{r}(Z, 1) \equiv 0 .
\end{aligned}
$$

From (30) follows, that practically $U_{z}(R, Z) \equiv 1, U_{r}(Z, R) \equiv 0$ (except for a thin boundary layer on the wall).

The presented theoretical analysis shows, that the using tangential input of the flows in the columns area lead to a significant decrease of the velocity radial non-uniformity and as a result an increase of the conversion degree in the column reactors.

\subsection{Simultaneous Mass and Heat Transfer Processes}

The heat and mass transfer kinetics theory shows [3], that the process rate depends on the characteristic velocity in the boundary layer. The big difference between these velocities in the cases of axial and tangential input of the flows $\left(\bar{u} \ll u_{\varphi}^{0}\right)$ leads to a substantial increase of the heat transfer rate through the column wall.

Let's consider a simultaneous mass and heat transfer processes in a column chemical reactor, where the velocity, concentration and temperature $t$ (deg) distributions in the column are denoted as:

$$
u=u(r), \quad c=c(r, z), \quad t(r, z) .
$$


The mass and heat transfer model in the physical approximations of the mechanics of continua [1]-[4] can be expressed as:

$$
\begin{aligned}
& u \frac{\partial c}{\partial z}=D\left(\frac{\partial^{2} c}{\partial z^{2}}+\frac{1}{r} \frac{\partial c}{\partial r}+\frac{\partial^{2} c}{\partial r^{2}}\right)-k c ; \\
& z=0, \quad c(r, 0) \equiv c_{0}, \quad \bar{u} c_{0} \equiv u c_{0}-D \frac{\partial c}{\partial z} ; \\
& r=0, \quad \frac{\partial c}{\partial r} \equiv 0 ; \quad r=r_{0}, \quad \frac{\partial c}{\partial r} \equiv 0 . \\
& u \frac{\partial t}{\partial z}=\frac{\lambda}{\rho c_{p}}\left(\frac{\partial^{2} t}{\partial z^{2}}+\frac{1}{r} \frac{\partial t}{\partial r}+\frac{\partial^{2} t}{\partial r^{2}}\right)+\frac{q}{\rho c_{p}} k c ; \\
& z=0, \quad t(r, 0) \equiv t_{0}, \quad \bar{u} t_{0} \equiv u t_{0}-\frac{\lambda}{\rho c_{p}} \frac{\partial t}{\partial z} ; \\
& r=r_{0}, \quad t \equiv t_{s} ; \quad-\lambda \frac{\partial t}{\partial r} \equiv k_{0},
\end{aligned}
$$

where $\rho\left(\mathrm{kg} \cdot \mathrm{m}^{-3}\right)$ is density, $c_{p}\left(\mathrm{~J} \cdot \mathrm{kg}^{-1} \cdot \mathrm{deg}^{-1}\right)$ - specific heat at constant pressure, $\lambda\left(\mathrm{J} \cdot \mathrm{m}^{-1} \cdot \mathrm{s}^{-1} \cdot \mathrm{deg}^{-1}\right)$ thermal conductivity, $q\left(\mathrm{~J} \cdot \mathrm{kg}^{-1}\right)$ 一heat effect of the chemical reaction, $k_{0}\left(\mathrm{~J} \cdot \mathrm{m}^{-2} \cdot \mathrm{s}^{-1}\right)$-local heat transfer flux. In the model ((32), (33)) $D, k, \lambda, \rho, c_{p}, q, k_{0}$ are temperature functions, where $t_{0} \leq t \leq t_{s}$ or $t_{s} \leq t \leq t_{0}$ in the case of endothermic $(q<0)$ or exothermic $(q>0)$ chemical reaction.

Practically the difference $\left|t_{0}-t_{s}\right|$ is not so big and in ((32), (33)) is possible to be used constant values of $D, k, \lambda, \rho, c_{p}, q, k_{0}$ at $t^{*}=\frac{t_{0}+t_{s}}{2}$.

From the condition $t_{s}=$ const. follows, that the volume heat generation in the column is equal to the interface heat transfer through the column wall:

$$
2 \pi \int_{0}^{r_{0}} r q k c \mathrm{~d} r=-2 \pi r_{0} \lambda\left(\frac{\partial t}{\partial r}\right)_{r=r_{0}},-\lambda\left(\frac{\partial t}{\partial r}\right)_{r=r_{0}}=\frac{r_{0} q k}{2} \bar{c}(z)=k_{0}(z) .
$$

A qualitative analysis of the model ((32), (33)) will be made, using generalized variables:

$$
\begin{aligned}
& r=r_{0} R, \quad z=l Z, \quad u(r)=u\left(r_{0} R\right)=\bar{u} U(R), \\
& c(r, z)=c\left(r_{0} R, l Z\right)=c_{0} C(R, Z), \\
& t(r, z)=t\left(r_{0} R, l Z\right)=t^{*} T(R, Z),
\end{aligned}
$$

where $r_{0}, l, \bar{u}, c_{0}, t^{*}$ are the characteristic (inherent) scales (maximal or average values) of the variables. The introducing the generalized variables (35) in ((32), (33)) leads to:

$$
\begin{gathered}
U(R) \frac{\partial C}{\partial Z}=\frac{D l}{\bar{u} r_{0}^{2}}\left(\frac{r_{0}^{2}}{l^{2}} \frac{\partial^{2} C}{\partial Z^{2}}+\frac{1}{R} \frac{\partial C}{\partial R}+\frac{\partial^{2} C}{\partial R^{2}}\right)-\frac{k l}{\bar{u}} C ; \\
Z=0, \quad C(R, 0) \equiv 1, \quad 1 \equiv U(R)-\frac{D}{\bar{u} l} \frac{\partial C}{\partial Z} ; \\
R=0, \quad \frac{\partial C}{\partial R} \equiv 0 ; \quad R=1, \quad \frac{\partial C}{\partial R} \equiv 0 . \\
U(R) \frac{\partial T}{\partial Z}=\frac{\lambda l}{\bar{u} \rho c_{p} r_{0}^{2}}\left(\frac{r_{0}^{2}}{l^{2}} \frac{\partial^{2} T}{\partial Z^{2}}+\frac{1}{R} \frac{\partial T}{\partial R}+\frac{\partial^{2} T}{\partial R^{2}}\right)+\frac{q l k c_{0}}{\bar{u} \rho c_{p} t^{*}} C ; \\
Z=0, \quad T(R, 0) \equiv T_{0}, \quad 1 \equiv U(R)-\frac{\lambda}{T_{0} \bar{u} \rho c_{p} l} \frac{\partial T}{\partial Z}, \quad T_{0}=\frac{t_{0}}{t^{*}} \\
R=1, \quad T \equiv T_{s} ; \quad \frac{\partial T}{\partial R} \equiv-\frac{r_{0}^{2} q k c_{0}}{2 \lambda t^{*}} \bar{C}(Z), \quad T_{s}=\frac{t_{s}}{t^{*}} .
\end{gathered}
$$


In the cases of very height column is possible to be used the approximation $0=\frac{r_{0}^{2}}{l^{2}} \leq 10^{-2}$ and the models ((36), (37)) are parabolic type. If average velocity $\bar{u}$ is very big, it is possible to be used the approximations $0=\frac{D l}{\bar{u} r_{0}^{2}} \leq 10^{-2}$ and $0=\frac{\lambda l}{\bar{u} \rho c_{p} r_{0}^{2}} \leq 10^{-2}$, i.e. the models ((33), (34)) are convective type:

$$
\begin{gathered}
U(R) \frac{\partial C}{\partial Z}=-\frac{k l}{\bar{u}} C ; \quad Z=0, \quad C(R, 0) \equiv 1 . \\
U(R) \frac{\partial T}{\partial Z}=\frac{q l k c_{0}}{\bar{u} \rho c_{p} t^{*}} C ; \quad Z=0, \quad T(R, 0) \equiv T_{0} .
\end{gathered}
$$

\subsection{Average Temperature Model}

The average temperature at the column cross-sectional area can be presented as

$$
\bar{t}(z)=\frac{2}{r_{0}^{2}} \int_{0}^{r_{0}} r t(r, z) \mathrm{d} r .
$$

The velocities and temperature distributions assume to be presented by the average functions ((8), (40)):

$$
u(r)=\bar{u} \tilde{u}(r), \quad t(r, z)=\bar{t}(z) \tilde{t}(r, z),
$$

where $\tilde{u}(r, z)$ and $\tilde{t}(r, z)$ represent the radial non-uniformity of both the velocity and the temperature distributions, satisfying the conditions:

$$
\frac{2}{r_{0}^{2}} \int_{0}^{r_{0}} r \tilde{u}(r) \mathrm{d} r=1, \quad \frac{2}{r_{0}^{2}} \int_{0}^{r_{0}} r \tilde{t}(r, z) \mathrm{d} r=1
$$

An average temperature model may be obtained if put the Expression (41) into the model Equation (33) and then multiply by $r$ and integrate with respect to $r$ over the interval $\left[0, r_{0}\right]$. The result is:

$$
\alpha_{t} \bar{u} \frac{\mathrm{d} \bar{t}}{\mathrm{~d} z}+\frac{\mathrm{d} \alpha_{t}}{\mathrm{~d} z} \bar{u} \bar{t}=\frac{\lambda}{\rho c_{p}} \frac{\mathrm{d}^{2} \bar{t}}{\mathrm{dz}^{2}} ; \quad z=0, \quad \bar{t}(0)=t_{0}, \quad \frac{\mathrm{d} \bar{t}}{\mathrm{~d} z}=0,
$$

where

$$
\alpha_{t}=\alpha_{t}(z)=\frac{2}{r_{0}^{2}} \int_{0}^{r_{0}} r \tilde{u} \tilde{d} d r
$$

A using of the generalized variables:

$$
Z=\frac{Z}{l}, \quad \bar{T}=\frac{\bar{t}}{t_{0}}, \quad \alpha_{t}(z)=\alpha_{t}(l Z)=A_{t}(Z),
$$

leads to:

$$
A_{t} \frac{\mathrm{d} \bar{T}}{\mathrm{~d} Z}+\frac{\mathrm{d} A_{t}}{\mathrm{~d} Z} \bar{T}=\frac{\lambda t_{0}}{\rho c_{p} \bar{u} l^{2}} \frac{\mathrm{d}^{2} T}{\mathrm{~d} Z^{2}} ; \quad Z=0, \quad \bar{T}=1, \quad \frac{\mathrm{d} \bar{T}}{\mathrm{~d} Z}=0 .
$$

Similar to (18) the function $A_{t}(Z)$ is possible to be obtained after solution of the problems (36) and (37):

$$
A_{t}(Z)=2 \int_{0}^{1} R U(R) \frac{T(R, Z)}{\bar{T}(Z)} \mathrm{d} R, \quad \bar{T}(Z)=\frac{\bar{t}(z)}{t^{*}}=2 \int_{0}^{1} R T(R, Z) \mathrm{d} R
$$

\section{Conclusions}

The solution of the modeling problem of the column chemical reactors is presented in the approximation of the mechanics of continua, where diffusion type of model and average concentration model are proposed for the qu- 
alitative and the quantitative analysis of the processes in column reactors, respectively.

The presented theoretical analysis shows that the conversion degree in the column chemical reactors decreases as a result of the radial non-uniformity of the axial velocity component.

A theoretical analysis of the simultaneous mass and heat transfer processes in column reactors is presented.

A method for substantially reducing the radial non-uniformity, and using a tangential introduction of the fluid in the column, is proposed as a utility model (application number 2464 column reactor for chemical processes 17 June 2013).

The main claims of the utility model are:

- a column apparatus for chemical reactions (column chemical reactor), characterized in that the fluid (reaction mixture) goes into the bottom of the column working zone directly and tangentially;

- a column apparatus for chemical reactions (column chemical reactor), characterized in that the radial nonuniformity of the velocity distribution over the cross-sectional area in the working zone of the column is minimized and the chemical processes rate is maximal;

- a column apparatus for chemical reactions (column chemical reactor), characterized in that the generated angular velocity component leads to an intensification of heat transfer through the walls of the column in the cases of the heating (cooling) of the reaction mixture.

\section{References}

[1] Boyadjiev, Chr. (2006) Diffusion Models and Scale-Up. International Journal of Heat and Mass Transfer, 49, 796799. http://dx.doi.org/10.1016/j.ijheatmasstransfer.2005.08.013

[2] Boyadjiev, Chr. (2009) Modeling of Column Apparatuses. Transactions of Academenergo, 3, 7-22.

[3] Boyadjiev, Chr. (2010) Theoretical Chemical Engineering. Modeling and Simulation. Springer-Verlag, Berlin Heidelberg, 594.

[4] Doichinova, M. and Boyadjiev, Chr. (2012) On the Column Apparatuses Modeling. International Journal of Heat and Mass Transfer, 55, 6705-6715. http://dx.doi.org/10.1016/j.ijheatmasstransfer.2012.06.079

[5] Lietuvos Respublika (1994) Patent No. 3884.

[6] Boyadjiev, Chr., Boyadjiev, B., Doichinova, M. and Popova-Krumova, P. (2013) Column Reactor for Chemical Processes. Utility Model, Application Number 2464. 